\title{
TO BE OR NOT TO BE: VERY YOUNG GLOBULAR CLUSTERS IN M31 ${ }^{1}$
}

\author{
Judith G. Cohen, ${ }^{2}$ Keith Matthews, ${ }^{3}$ and P. Brian Cameron ${ }^{2}$ \\ Received 2005 September 2; accepted 2005 October 7; published 2005 November 1
}

\begin{abstract}
We present observations made with the newly commissioned Keck laser guide star adaptive optics (LGSAO) system of six objects in M31 that are alleged in multiple recent studies to be young globular clusters (GCs); all are supposed to have ages $\leq 5 \mathrm{Gyr}$. The resulting FWHM of the PSF core in our images is $\sim 70$ mas. The four youngest of these objects are asterisms; they are with certainty not young GCs in M31. Based on their morphology, the two oldest are GCs in M31. While the M31 GCs with ages 5-8 Gyr appear to be mostly genuine, it appears that many of the alleged very young GCs in M31 are spurious identifications. This problem will be even more severe in studies now underway of the GC systems of more distant spiral galaxies, for which imaging at the spatial resolution of our observations in M31 may not be adequate to detect sample contamination by asterisms.
\end{abstract}

Subject headings: galaxies: individual (M31) — galaxies: star clusters

\section{INTRODUCTION}

M31 is the nearest large galaxy to our own, and as such plays a crucial role in many areas of extragalactic astronomy, both in its own right and as a test bed for techniques for studying more distant systems. Early studies of its globular cluster system (GCS; see, e.g., Frogel et al. 1980), suggested that the M31 GCS is very similar to our own, except that it contains more members ( $\sim 350$ vs. $\sim 150$ members) and extends to higher metallicity than does our own GCS, whose properties are reviewed by Harris (1996). In this view, essentially all of the M31 globular clusters (GCs) are old, with typical ages of 10$12 \mathrm{Gyr}$, as is true of our own GCS; see, e.g. Rosenberg et al. (1999) and De Angeli et al. (2005).

Recently, as more of the members of the M31 GCS have been studied and additional spectra of these objects have been obtained, there have been several papers asserting the existence of many very young (age $\leq 1 \mathrm{Gyr}$ ), young (age 1-2 Gyr), and intermediate age (3-6 Gyr) GCs in M31, in addition to a substantial population of old GCs. Among these studies, which discuss to varying degrees the possibility of sample contamination, are the works of Barmby et al. (2000), Beasley et al. (2005, hereafter Bea05), Puzia et al. (2005b, hereafter P05), Burstein et al. (2004, hereafter Bur04), and Fusi Pecci et al. (2005, hereafter F05). Color-magnitude diagrams for a few of the brightest M31 GCs have been obtained from Hubble Space Telescope (HST) imaging; see, e.g., Rich et al. (2005) and Williams \& Hodge (2001). The latter focused on very young GCs, studying four of them in detail. Additional recent papers offer more sophisticated interpretations of the presence of numerous very young and populous clusters in M31 (e.g., Lee \& Worthey 2005), using these results to derive characteristics of the formation of M31.

A young globular cluster is here defined as a cluster of stars of essentially the same age and initial chemical composition that has a total stellar mass similar to that of the Galactic GCs. It must be centrally concentrated and have a projected shape

\footnotetext{
${ }^{1}$ Based in part on observations obtained at the W. M. Keck Observatory, which is operated as a scientific partnership among the California Institute of Technology, the University of California, and the National Aeronautics and Space Administration.

${ }^{2}$ Palomar Observatory, MS 105-24, California Institute of Technology, Pasadena, CA 91125; jlc@astro.caltech.edu,pbc@astro.caltech.edu.

${ }^{3}$ California Institute of Technology, Downs Laboratory, MS 320-47, Pasadena, CA 91125; kym@caltech.edu.
}

similar to that of Galactic GCs. It is an object that, with the passage of time, when it reaches an age of $10 \mathrm{Gyr}$ would resemble a typical Galactic GC. Such populous young clusters are not found in our Galaxy, while F05 claim that the youngest of these comprise $15 \%$ of the whole of the M31 GCS.

In the present Letter we show, from an admittedly small sample of objects, that $2 / 3$ of the sample of purported very young and young GCs in M31 we observed turned out not to be GCs in M31. It is thus likely that many of the alleged youngest GCs in M31 are asterisms rather than globular clusters.

\section{OBSERVATIONS}

We used the newly commissioned laser guide star assisted adaptive optics (LGSAO; Wizinowich et al. 2004) at the Keck II $10 \mathrm{~m}$ telescope on Mauna Kea, Hawaii. The Near Infrared Camera 2 (NIRC2; PI: K. Matthews) was used with a $K^{\prime}$ filter in the narrow field mode. Each pixel is then 0"010 on a side, and the field is approximately $10^{\prime \prime} \times 10^{\prime \prime}$. This requires a natural tip/tilt correction star which for best resulting image quality must have $R<16.5$ mag and be located less than $45^{\prime \prime}$ from the object. The LGS Web page ${ }^{4}$ provides details on the system performance, including the image degradation when these requirements are not met. To be conservative, we always obeyed these restrictions in designing our program.

LGSAO has the characteristic that the resulting images have a Strehl ratio somewhat less than that of the best ratio achieved with natural guide star adaptive optics (AO) on-axis, but have a much more uniform PSF over the whole field than does natural guide star AO, where the PSF degrades rapidly as one moves away from the correction star. LGSAO permits the use of fainter guide stars (required only for the tip/tilt correction), which can be located somewhat farther from the object of interest than cans natural guide star AO (Roggemann \& Welsh 1996).

We observed for $4 \mathrm{hr}$ on each of 2 nights in 2005 August. These nights were marked by various technical problems, mostly at the beginning of each night, and by a shutdown of the laser for several hours at the request of the US Space Command. However, once the laser was working, it ran without a major fault throughout each night. The natural seeing ranged from $0 " 8-1 " .5$ during the 2 nights. The NIRC2 exposures were

\footnotetext{
${ }^{4}$ See http://www2.keck.hawaii.edu/optics/lgsao/.
} 
100-150 s each, such long exposures in the thermal IR being feasible with such a large telescope only because of the very small pixel scale and resulting very low sky background signal. The longest total integration time was $1650 \mathrm{~s}$. Individual images show a PSF even better than that of $H S T$, with a core of between 60 and 80 mas. The first diffraction ring is clearly visible on the best of these images. There is also a diffuse halo component in the PSF, which for present purposes we ignore.

The LGSAO system has an acquisition camera with a field of $2^{\prime}$ operating in the optical (effectively Johnson $R$ ). For each object, the pointing for the tip/tilt star was checked with this camera, then an offset to the object of interest of less than 45" was made. We used the first few integrations on target to identify and center up the object, then ran a script written by D. Thompson which made random moves within a specified box size to acquire the subsequent images. This moved the object around the field, allowing a determination of the sky background from the integrations on the object without separate sky exposures. Dome flats and bias frames were also acquired. Since all our objects were within $3^{\circ}$ of each other, the move to and acquisition of subsequent targets was accomplished within 10 minutes.

We followed standard reduction procedures for the set of images of each object. Each frame was flat-fielded, backgroundsubtracted, and repaired for bad pixels. We then produced a mask for each exposure to remove all detected objects. We combined these masked images to produce an improved sky frame for each of the six science targets for a second round of sky subtraction. The optical distortion of the NIRC2 narrow field of view camera is relatively small. It was corrected using algorithms derived from the preshipment review documents ${ }^{5}$ with the IDL procedure provided by the Keck Observatory. ${ }^{6}$ We then shifted, co-added, and trimmed the set of exposures for each object, then divided by the number of frames to produce the final images shown in Figure 1.

\section{SAMPLE SELECTION}

We compiled a list of all GCs in M31 with ages less than 6 Gyr from the studies of Bea05, Bur04 and P05. (The very recent extensive study of F05 was not available when this was being done.) We then applied the restrictions for the LGS tip/ tilt star described above. We used the USNO B1.0 Catalog (Monet et al. 2003) to get a list of potential tip/tilt stars with astrometric-quality positions. Each such star (and each object) was then checked on the Digitized Sky Survey I frames to make sure that each tip/tilt star was real, not a galaxy, and that it looked stellar. We also eliminated objects with an obvious large spatially varying background in the vicinity of the tip/ tilt star (and the object) due to concern over the stability of the LGSAO correction.

Object coordinates and photometry were taken from the recent update of the Bologna catalog of M31 GCs of Galleti et al. (2004). Each of the GCs observed was classified as a certain GC in M31 (classification flag 1) in this catalog, and each has a radial velocity consistent with membership in M31.

Our initial list of candidates from these three recent papers contained $24 \mathrm{GCs}$. After the requirements described above were imposed, eight objects remained. The six youngest of these were observed. The ages of the two GCs in the selected sample suitable for LGSAO imaging at the Keck Telescope that were

\footnotetext{
${ }^{5}$ Available at http://alamoana.keck.hawaii.edu/inst/nirc2/.

${ }^{6}$ See http://www2.keck.hawaii.edu/optics/lgsao/software/index.html.
}

not observed are 6.6 and 7.1 Gyr, older than any of those we observed.

The properties of our sample of M31 GCs observed with LGSAO are given in Table 1, including their distances from the center of M31 along the major $(X)$ and minor $(Y)$ axes of this galaxy.

\section{RESULTS}

Figure 1 shows the resulting $K^{\prime}$ images taken with LGSAO of the six very young or young GCs in M31. It is immediately apparent that only two of these are genuine GCs in M31. These correspond to the two objects in our sample with the oldest ages. Among the youngest objects in our admittedly small sample, four of the putative M31 GCs are clearly asterisms in M31; they definitely are not genuine M31 GCs. Two of these (B314 and B380) appear to be very faint associations or clusters in M31 with superposed much brighter objects. These are not genuine M31 GCs; their integrated light as detected by normal seeing-limited ground-based observations would be dominated by the superposed stars rather than by the very faint association/ cluster, and their spatial distributions are not sufficiently centrally concentrated. In all cases, the spurious objects as seen with a spatial resolution typical of seeing-limited ground-based optical observations would appear to be slightly extended objects. It is not surprising that they were picked up as GCs in M31.

The HST archive was searched for the 24 objects in our original sample prior to filtering for LGSAO. Wide Field Planetary Camera 2 (WFPC2) images that included five of these were located. One (B210, not in our sample due to the lack of a suitable laser guide star) appears to be an asterism rather than a GC in M31. The other four that were found there (B232, B311, B315, and B324), all of which have an age $>3 \mathrm{Gyr}$, appear to be GCs in M31. One of these four (B232) is in our LGSAO sample; it has the appearance of a GC in M31 there as well. Williams \& Hodge (2001) presented HST images for four "young" M31 GCs. Ignoring duplications, they thus verified that in addition B319, B342, and B368 are also genuine M31 GCs. Racine (1991, hereafter R91) imaged 109 halo candidate M31 GCs with the Canada-France-Hawaii Telescope (CFHT) with 0"7 FWHM seeing to eliminate interlopers.

\section{IMPLICATIONS}

With the high spatial resolution of LGSAO at the Keck II Telescope we have found that a large fraction of the putative very young and young GCs we observed are not GCs in M31. This suggests that many of the youngest GCs in M31 are asterisms rather than M31 GCs. Thus, all the theoretical papers explaining the surprising presence of numerous very young GCs in M31 or using such to reach inferences about the formation of M31 must be reconsidered, as must the kinematic analyses based on the M31 GC radial velocities such as Morrison et al. (2004).

The initial reconnaissance of the M31 GCS began more than 70 years ago with Hubble (1932). In recent years people have concentrated on detailed studies of larger samples of GCs in M31. Recently Bea05, P05, Bur04, and F05, among others, have claimed the presence of a substantial number of very young GCs in M31. In photometric studies of the M31 GCS, outliers that appear too red (i.e., older than $14 \mathrm{Gyr}$ ) are rejected as distant background galaxies, but outliers that appear very blue (i.e., very young) are usually not rejected. Also, the issue of contamination of samples of GCs with asterisms becomes 

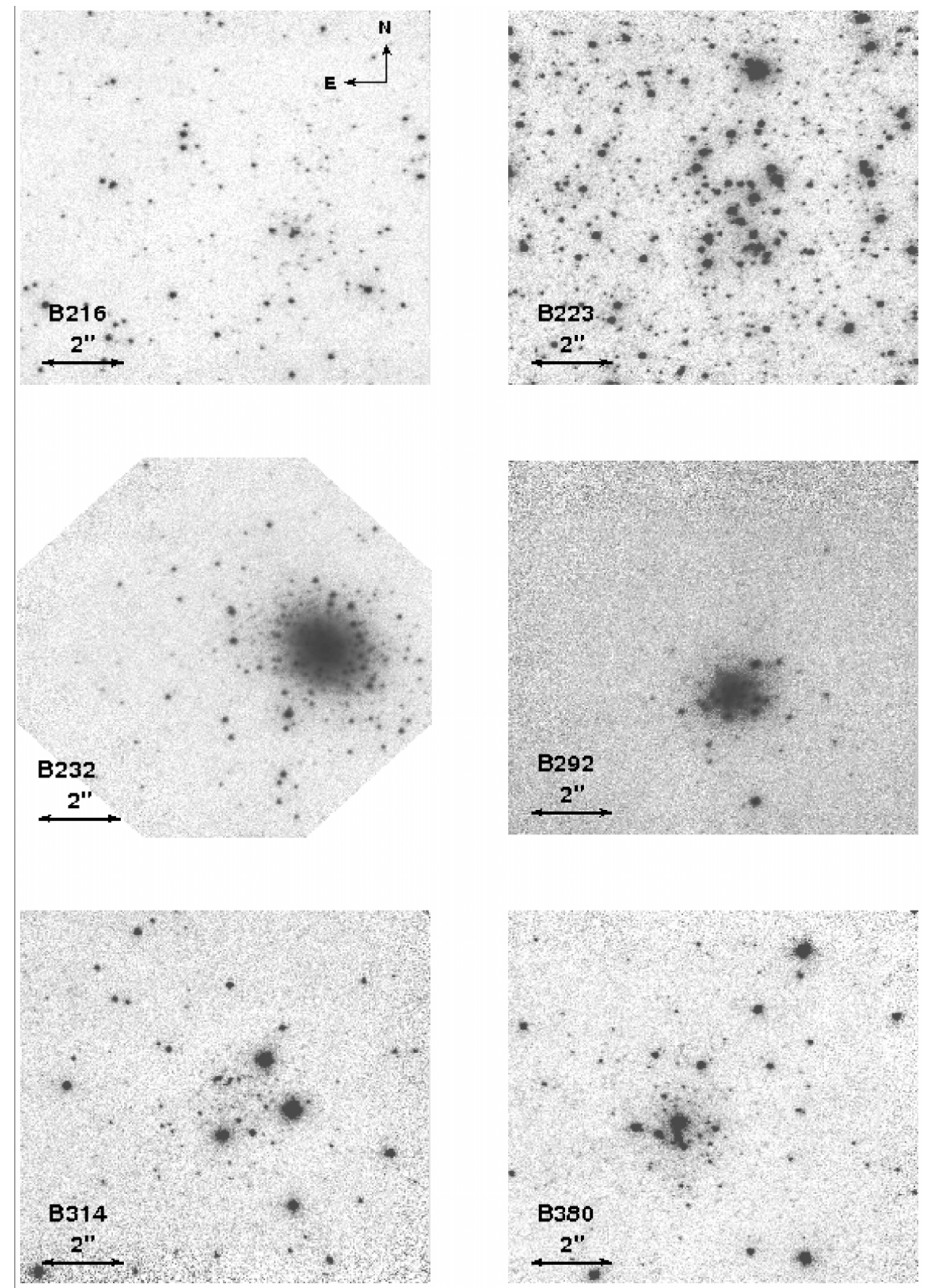

FIG. 1.-LGSAO images from the Keck telescope are shown for six putative very young or young globular clusters in M31. Based on their morphology, only two of these are actually GCs in M31. These are $K^{\prime}$ images all oriented as indicated in the first panel. The field shown for each is approximately $10^{\prime \prime}$ on a side with a pixel scale of $0^{\prime \prime} 010$ pixel $^{-1}$ and FWHM with a core of 60-80 mas.

more acute as one presses fainter. While the claims of middleaged (5-8 Gyr) GCs in M31 are not challenged by our observations, if there are no or few very young M31 GCs, then at least some of the purported middle- aged GCs might easily be the outliers of the M31 GCS, once the sample is restricted only to valid GCs in this galaxy.
Cohen et al. $(1998,2003)$ found that there are no globular clusters with ages $\$ 8 \mathrm{Gyr}$ in the Virgo gE galaxies M87 and NGC 4472, a result extended by Puzia et al. (2005a) to less luminous early-type galaxies with a somewhat looser minimum age of 5 Gyr. Elliptical galaxies have smooth surface brightness profiles, at least outside their nuclei, and hence the study of 
TABLE 1

Sample of M31 Globular Clusters Imaged with LGSAO

\begin{tabular}{|c|c|c|c|c|c|c|}
\hline ID & $\begin{array}{c}V, B-V^{\mathrm{a}} \\
(\mathrm{mag})\end{array}$ & $\begin{array}{c}v_{r}^{\mathrm{b}} \\
\left(\mathrm{km} \mathrm{s}^{-1}\right)\end{array}$ & $\begin{array}{l}\text { Age } \\
(\mathrm{Gyr})\end{array}$ & References $^{\mathrm{c}}$ & $\begin{array}{c}X, Y^{\mathrm{d}} \\
(\operatorname{arcmin})\end{array}$ & Comments \\
\hline B223. & $15.81,0.15$ & -86 & 0.5 & Bar00, J03, Bur04 & $+26.4,-3.8$ & Asterism \\
\hline B216 . & $17.45,0.20$ & -96 & 0.5 & Bar00, J03, Bur04 & $+26.9,+0.9$ & Asterism \\
\hline B314 & $17.63,0.69$ & -318 & $1.0 \pm 0.1$ & Bar00, Bea04 & $-69.9,-10.8$ & Asterism $^{\mathrm{e}}$ \\
\hline B380 & $17.12,1.01$ & -121 & $1.0 \pm 0.1$ & Bar00, Bea04, Bur04 & $+58.5,-2.1$ & Asterism $^{\mathrm{f}}$ \\
\hline B232 & $15.67,0.72$ & -151 & $2-5$ & Bur04 & $+12.5,-17.5$ & M31 GC, WFPC $2^{\mathrm{t}}$ \\
\hline B292 & $17.00,0.89$ & -331 & $2.7-5.9$ & R91, Bar00, Bea04, Bea04 & $-58.3,47.4$ & M31 GC \\
\hline
\end{tabular}

${ }^{a}$ From Galleti et al. (2004).

${ }^{\mathrm{b}}$ From Huchra et al. (1991), Galleti et al. (2004), or Beasley et al. (2004).

${ }^{\mathrm{c}}$ Reference codes are as follows: Bar00 = Barmby et al. 2000; Bea04 = Beasley et al. 2004; Bea05 = Beasley et al. 2005; Bur04 = Burstein et al. 2004; J03 = Jiang et al. 2003; R91 = Racine 1991.

${ }^{\mathrm{d}}$ The coordinate system defined by Huchra et al. (1991) is used.

${ }^{\mathrm{e}}$ Very faint loose association/cluster with superposed much brighter stars.

${ }^{\mathrm{f}} H S T$ image taken 1998 September as part of an archive program for parallel WFPC2 images in the neighborhood of bright galaxies, PI: S. Casertano.

their GC systems does not suffer from the problem discussed here for the M31 GCS.

The potential confusion of GCs with asterisms is bound to become more serious as the GC systems of more distant spiral galaxies are explored. Such projects are already underway by several groups at the largest ground-based telescopes. Even the spatial resolution offered by $\mathrm{AO}$ on a $10 \mathrm{~m}$ ground-based telescope will at some point no longer be sufficient to eliminate this possibility. Outliers may result from large observational errors caused by insufficient integration time for such faint objects or from sample contamination much larger than previously suspected. An example of the former is the claim for young GCs in the Virgo elliptical NGC 4365 by Larsen et al. (2003), which, with better data, have subsequently been shown to be old (Brodie et al. 2005), while the present Letter illustrates the importance of the latter issue. Caution is urged in interpreting the results of such studies.

We congratulate the Keck LGSAO team on the recent suc- cessful commissioning of the LGSAO system. The entire Keck user community owes a huge debt to Jerry Nelson, Gerry Smith, and many other people who have worked to make the Keck Telescope a reality and to operate and maintain the Keck Observatory. We are grateful to the W. M. Keck Foundation for the vision to fund the construction of the W. M. Keck Observatory. The authors wish to extend special thanks to those of Hawaiian ancestry on whose sacred mountain we are privileged to be guests. Without their generous hospitality, none of the observations presented here would have been possible. This research has made use of the USNOFS Image and Catalog Archive operated by the US Naval Observatory, Flagstaff Station (http://www.nofs.navy.mil/data/fchpix/) and the Digitized Sky Surveys produced at STScI based on photographic data obtained using the Oschin Schmidt Telescope on Palomar Mountain and the UK Schmidt Telescope. We are grateful to the National Science Foundation for partial support under grant AST-0205951 to J. G. C.

\section{REFERENCES}

Barmby, P., Huchra, J. P., Brodie, J. P., Forbes, D. A., Schroder, L. L., \& Grillmair, C. J. 2000, AJ, 119, 727

Beasley, M. A., et al. 2004, AJ, 128, 1623

. 2005, AJ, 129, 1412 (Bea05)

Brodie, J. P., et al. 2005, AJ, 129, 2643

Burstein, D., et al. 2004, ApJ, 614, 158 (Bur04)

Cohen, J. G., Blakeslee, J. P., \& Côté, P. 2003, ApJ, 592, 866

Cohen, J. G., Blakeslee, J. P., \& Ryzhov, A. 1998, ApJ, 496, 808

De Angeli, F., et al. 2005, AJ, in press

Frogel, J. A., Persson, S. E., \& Cohen, J. G. 1980, ApJ, 240, 785

Fusi Pecci, F., Bellazzini, M., Buzzoni, A., De Simone, E., Federici, L., \& Galleti, S. 2005, AJ, 130, 554 (F05)

Galleti, S., Federici, L., Bellazzini, M., Fusi Pecci, F., \& Macrina, S. 2004, A\&A, 416, 917

Harris, W. 1996, AJ, 112, 1487

Hubble, E. P. 1932, ApJ, 76, 44

Huchra, J. P., Brodie, J. P., \& Kent, S. M. 1991, ApJ, 370, 495
Jiang, L., Ma., J., Zhou, X., Chen, J., Wu, H., \& Jiang, Z. 2003, AJ, 125, 727 Larsen, S. S., Brodie, J. P., Beasley, M. A., Forbes, D. A., Kissler-Patig, M., Kuntschner, H., \& Puzia, T. H. 2003, ApJ, 585, 767

Lee, H., \& Worthey, G. 2005, ApJS, 160, 176

Monet, D. G., et al. 2003, AJ, 125, 984

Morrison, H. L., Harding, P., Perrett, K., \& Hurley-Keller, D. 2004, ApJ, 603, 87

Puzia, T. H., Kissler-Patig, M., Thomas, D., Maraston, C., Saglia, R. P., Bender, R., Goudfrooij, P., \& Hempel, M. 2005a, A\&A, 439, 997

Puzia, T. H., Perrett, K. M., \& Bridges, T. J. 2005b, A\&A, 434, 909 (P05)

Racine, R. 1991, AJ, 101, 865 (R91)

Rich, R. M., et al. 2005, AJ, 129, 2670

Roggemann, M. C., \& Welsh, B. 1996, Imaging through Turbulence (Boca Raton: CRC Press), 175

Rosenberg, A., Saviane, I., Piotto, G., \& Aparicio, A. 1999, AJ, 118, 2306

Williams, B. F., \& Hodge, P. W. 2001, ApJ, 548, 190

Wizinowich P. L., et al. 2004, Proc. SPIE, 5490, 1 